\title{
The Asymptotics of the Ray-Singer Analytic Torsion Associated with High Powers of a Positive Line Bundle
}

\author{
Jean-Michel Bismut ${ }^{1, \star}$ and Eric Vasserot ${ }^{2}$ \\ ${ }^{1}$ Département de Mathématique, Université Paris-Sud, F-91405 Orsay, France \\ ${ }^{2}$ Ecole Normale Supérieure, 45 rue d'Ulm, F-75005 Paris, France
}

\begin{abstract}
The purpose of this paper is to establish an asymptotic fomula for the Ray-Singer analytic torsion associated with increasing powers of a given positive line bundle.
\end{abstract}

Let $M$ be a compact complex manifold, equipped with a smooth Hermitian metric. Let $\xi$ be a holomorphic Hermitian vector bundle, and let $\mu$ be a positive Hermitian line bundle. For $p \in N$, let $E_{p}$ be a holomorphic Hermitian flat vector bundle on $M$. By the Kodaira vanishing theorem, for $p$ large enough, the sheaf $\mathcal{O}_{M}\left(\mu^{\otimes p} \otimes \xi \otimes E_{p}\right)$ has cohomology only in degree zero. We first equip $\mu$ with a metric whose curvature is positive. Let $\tau_{p}$ be the Ray-Singer analytic torsion [RS] of the Dolbeault complex on $\mu^{\otimes p} \otimes \xi \otimes E_{p}$. In this paper, we establish an asymptotic formula for $\log \left(\tau_{p}\right)$ as $p \rightarrow+\infty$. To prove this formula, we essentially use results of Bismut [B1], where the asymptotic Morse inequalities of Demailly [D1] were established by using a heat equation method. We also establish an asymptotic bound for $\log \left(\tau_{p}\right)$ when the metric on $\mu$ is arbitrary.

This work was partly motivated by a lecture given in February 1988 by Y. Miyaoka on his current research on analytic torsion. Our asymptotic results may have useful applications in the theory of determinant bundles of direct images and their Quillen metrics, along the lines of Quillen [Q] and Bismut-Gillet-Soulé [BGS2]. In view of a result of Bost [Bo], which relates Quillen metrics to metrics introduced in Faltings [F], our asymptotic formula refines an asymptotic result of Faltings [F, Theorem 2]. It has been recently used by Gillet and Soule in [GS] to establish ampleness results for arithmetic manifolds, and also by Vojta [V].

Our paper is organized as follows. In Sects. a)-e), we assume that the metric on the positive line bundle $\mu$ has positive curvature. In a) we give our main assumptions and notations. In b) we establish a key estimate for the lowest eigenvalue of the Hodge Laplacian associated with the Hermitian vector bundle $\mu^{\otimes p} \otimes \xi \otimes E_{p}$. In c),

\footnotetext{
* This paper was written while the first author was visiting the Institut des Hautes Etudes Scientifiques (Bures-sur-Yvette) during the academic year 1987-1988
} 
we recall the results of [B1] concerning the asymptotics of certain heat kernels as $p \rightarrow+\infty$, and we derive asymptotic formulas on linear combinations of traces of such heat kernels. In $d$ ), we establish our asymptotic formula for $\log \left(\tau_{p}\right)$. In e), we give an application of this formula to asymptotic anomaly formulas on the $L_{2}$ metric of the determinant line $\operatorname{det} H^{0}\left(M, \mu^{\otimes p} \otimes \xi \otimes E_{p}\right)$. In f), we obtain an asymptotic bound for $\log \left(\tau_{p}\right)$ when the metric on $\mu$ is arbitrary.

The results contained in this paper were announced in [BV].

\section{a) Assumptions and Notations}

Let $M$ be a compact connected complex manifold of complex dimension $l$. Let $T M$ be the complex tangent space to $M$.

Let $\left(\mu,\|\|_{\mu}\right)$ be a holomorphic Hermitian positive line bundle on $M$. If $\nabla^{\mu}$ is the holomorphic Hermitian connection on $\left(\mu,\|\|_{\mu}\right)$ and if $r$ is the curvature of $\nabla^{\mu}$, positivity here means that if $X \in T M, X \neq 0$, then $r(X, \bar{X})>0$.

Let $\xi$ be a holomorphic vector bundle on $M$. We assume that $\xi$ is equipped with a Hermitian metric \|\|$_{\xi}$. Let $\nabla^{\xi}$ the corresponding holomorphic Hermitian connection on $\xi$ and let $L^{\xi}$ be its curvature. Let $E$ be a holomorphic Hermitian flat vector bundle on $M$. Then if $\nabla^{E}$ is the holomorphic Hermitian connection on $E$, the curvature of $\nabla^{E}$ vanishes.

We now take $p \in N$. For $0 \leqq q, q^{\prime} \leqq l$, let $\Omega^{\left(q, q^{\prime}\right)}\left(\mu^{\otimes p} \otimes \xi \otimes E\right)$ be the set of smooth sections of $\Lambda^{\left(q, q^{\prime}\right)}\left(T^{*} M\right) \otimes \mu^{\otimes} \otimes \xi \otimes E$ on $M$. Let $\bar{\partial}_{p}$ be the Dolbeault operator acting on the Dolbeault complex $\Omega^{(0, .)}\left(\mu^{\otimes p} \otimes \xi \otimes E\right)$.

We equip $\mu^{\otimes p}$ with the metric \|\|$_{\mu}^{\otimes p}$. So $\mu^{\otimes p} \otimes \xi \otimes E$ is a holomorphic Hermitian vector bundle on $M$. We note by $\langle,\rangle_{p}$ the Hermitian product on $\mu^{\otimes p} \otimes \xi \otimes E$. Let \|\|$_{T M}$ be a smooth Hermitian metric on $T M$, and let $\langle,\rangle_{T M}$ be the corresponding Hermitian product. Let $d x$ denote the corresponding volume form on $M . d x$ will be considered as a $(l, l)$ form on $M$.

Let $J$ be the complex structure of $T_{R} M . \omega$ denotes the Kähler form on $T_{R} M$,

$$
X, Y \rightarrow \omega(X, Y)=\langle X, J Y\rangle_{T M}
$$

Let $*$ be the star operator associated with \|\|$_{T M}[\mathrm{GrH}, \mathrm{p}$. 82]. We equip $\Omega^{(0, .)}\left(\mu^{\otimes p} \otimes \xi \otimes E\right)$ with the Hermitian product

$$
\alpha, \alpha^{\prime} \in \Omega^{(0, .)}\left(\mu^{\otimes p} \otimes \xi \otimes E\right) \rightarrow\left\langle\alpha, \alpha^{\prime}\right\rangle=\int_{M}\left\langle\alpha,{ }^{*} \alpha^{\prime}\right\rangle_{p} .
$$

Let $\bar{\partial}_{p}^{*}$ be the formal adjoint of $\bar{\partial}_{p}$ with respect to the Hermitian product (2). Set

Equivalently

$$
D_{p}=\bar{\partial}_{p}+\bar{\partial}_{p}^{*}, \quad \bar{\square}_{p}=\left(D_{p}\right)^{2} \text {. }
$$

$$
\bar{\square}_{p}=\bar{\partial}_{p} \bar{\partial}_{p}^{*}+\bar{\partial}_{p}^{*} \bar{\partial}_{p} .
$$

Let $\left\{H^{q}\left(M, \mu^{\otimes p} \otimes \xi \otimes E\right)\right\}_{0 \leqq q \leqq l}$ be the cohomology groups of the complex $\left(\Omega^{(0, .)}\left(\mu^{\otimes p} \otimes \xi \otimes E\right), \bar{\partial}_{p}\right)$. By a form of Kodaira's vanishing theorem [GrH, p. 159], we know that there exists $p_{0} \in N$, such that for any $p \geqq p_{0}$,

$$
H^{q}\left(M ; \mu^{\otimes p} \otimes \xi \otimes E\right)=0, \quad q \geqq 1 .
$$


Let $\bar{\square}_{p}^{q}$ be the restriction of $\bar{\square}_{p}$ to $\Omega^{(0, q)}\left(\mu^{\otimes p} \otimes \xi \otimes E\right)$. By Hodge theory, we deduce from (5) that for $p \geqq p_{0}, q \geqq 1, \bar{\square}_{p}^{q}$ is an invertible operator.

\section{b) The Lowest Eigenvalue of $\bar{\square}_{p}^{q}$}

We now prove a refined version of Kodaira's vanishing theorem.

Theorem 1. For $p \in N, 0 \leqq q \leqq l$, let $\lambda_{p}^{q}$ be the lowest eigenvalue of the operator $\bar{\square}_{p}^{q}$ acting on $\Omega^{(0, q)}\left(\mu^{\otimes p} \otimes \xi \otimes E\right)$. There exist constants $c>0, c^{\prime}>0$ not depending on $E$, which are such that for $p \in N, q \geqq 1$,

$$
\lambda_{p}^{q} \geqq c p-c^{\prime} .
$$

Proof. We proceed as in the proof in [GrH, p. 160] of the asymptotic vanishing theorem of Kodaira. Let $K_{M}=\operatorname{det}\left(T^{*} M\right)$ be the canonical bundle on $M$, and let $K_{M}^{*}=\operatorname{det}(T M)$ be its dual. We equip $K_{M}, K_{M}^{*}$ with the metrics induced by the metric of $T M$, and with the corresponding holomorphic Hermitian connections.

Since $K_{M}, K_{M}^{*}$ are holomorphic Hermitian line bundles which are such that $K_{M} \otimes K_{M}^{*}$ is canonically trivial, by comparing the corresponding $\bar{\partial}$ operators, one easily verifies that for any $p \in N, 0 \leqq q \leqq l$, the Laplacian $\bar{\square}_{p}^{q}$ acting on $\Omega^{(0, q)}\left(\mu^{\otimes p} \otimes \xi \otimes E\right)$ has the same spectrum as the Laplacian $\bar{\square}_{p}^{\prime q}$ acting on $\Omega^{(l, q)}\left(\mu^{\otimes p} \otimes \xi \otimes E \otimes K_{M}^{*}\right)$.

Set $\tilde{\xi}=\xi \otimes K_{M}^{*}, \tilde{\xi}^{\prime}=\tilde{\xi} \otimes E$. Let $L^{\tilde{\xi}}$ be the curvature of the holomorphic Hermitian connection on $\widetilde{\xi}$.

Let $w_{1}, \ldots, w_{l}$ be an orthonormal base of $T M$, and let $w^{1}, \ldots, w^{l}$ be the dual base of $T^{*} M . \bar{w}_{1}, \ldots, \bar{w}_{l}$ and $\bar{w}^{1}, \ldots, \bar{w}^{l}$ denote the corresponding conjugate bases. Set

$$
\Lambda=-\sqrt{-1} \sum_{1}^{l} i_{\bar{w}_{j}} i_{w_{j}}, \quad N_{p}=L^{\tilde{\xi}} \otimes I_{E}+\operatorname{pr} I_{\tilde{\xi}^{\prime}} .
$$

$N_{p}$ will be considered as a section of $\Lambda^{(1,1)}\left(T^{*} M\right) \otimes \operatorname{End}\left(\tilde{\xi}^{\prime}\right)$. By the generalized Bochner-Kodaira-Nakano formula of Demailly [D2, Theorem 0.3], we know that there exists an elliptic second order nonnegative operator $\square_{p}^{\prime}$ and an operator $T$ of order 0 acting on $\Lambda^{(\ldots)}\left(T^{*} M\right)$ and not depending on $p$ or $E$ such that

$$
\bar{\square}_{p}^{\prime}=\square_{p}^{\prime}+i\left[N_{p}, \Lambda\right]+T \otimes 1_{\tilde{\xi}^{\prime}} .
$$

Take $x \in M$. We may and we will assume that for $i \neq j, r_{x}\left(w_{i}, \bar{w}_{j}\right)=0$. Then one easily verifies that at $x$

$$
i[r, \Lambda]=\sum_{1}^{l} r\left(w_{j}, \bar{w}_{j}\right)\left[w^{j} \wedge i_{w_{j}}+\bar{w}^{j} \wedge i_{\bar{w}_{j}}-1\right] .
$$

There exists $c>0$ such that for any $x \in M$, and any $X \in T M,\|X\|_{T M}=1$, then $r(X, \bar{X}) \geqq c$. Also if $\alpha \in \Omega^{(l, q)}\left(\mu^{\otimes p} \otimes \tilde{\xi}^{\prime}\right)$, we deduce from (9) that

$$
\langle i[r, \Lambda] \alpha, \alpha\rangle \geqq c q\langle\alpha, \alpha\rangle \text {. }
$$

Clearly, there exists $C>0$ such that

$$
\left\|\left[L^{\tilde{\xi}}, \Lambda\right]\right\|+\|T\| \leqq C .
$$


Finally $\square_{p}^{\prime}$ is a nonnegative non-operator. From (8)-(11), we deduce that if $\alpha \in \Omega^{(l, q)}\left(\mu^{\otimes p} \otimes \tilde{\xi}^{\prime}\right)$, then

$$
\left\langle\bar{\square}_{p}^{\prime q} \alpha, \alpha\right\rangle \geqq(c p q-C)\langle\alpha, \alpha\rangle .
$$

So if $\lambda_{p}^{\prime q}$ is the lowest eigenvalue of $\bar{\square}_{p}^{\prime q}$, we find from (12) that

$$
\lambda_{p}^{\prime q} \geqq c p q-\mathrm{C} .
$$

Since $\lambda_{p}^{q}=\lambda_{p}^{\prime q},(6)$ follows from (13).

\section{c) The Asymptotic of the Heat Kernels as $\boldsymbol{p} \rightarrow+\infty$}

We assume that the flat bundle $E$ itself depends on $p$, and will now be denoted $E_{p}$. We will otherwise keep the notations of Sects. a) and b).

For $p \in N, 0 \leqq q \leqq l, t>0$, let $\operatorname{Tr}\left[\exp \left(-t \bar{\square}_{p}^{q}\right)\right]$ be the trace of the operator $\exp \left(-t \bar{\square}_{p}^{q}\right)$. For any $p \in N, 0 \leqq q \leqq l, k \in N$, as $t \downarrow 0$, we have the asymptotic expansion

$$
\frac{1}{r k\left(E_{p}\right)} p^{-l} \operatorname{Tr}\left[\exp \left(-\frac{t}{p} \bar{\square}_{p}^{q}\right)\right]=\sum_{j=-l}^{k} a_{p, j}^{q} t^{j}+o\left(t^{k}\right) \text {. }
$$

Of course, since $E_{p}$ is flat, $a_{p, j}^{q}$ does not depend on $E_{p}$.

Let $w_{1}, \ldots, w_{l}$ be an orthonormal base of $T M$, let $w^{1}, \ldots, w^{l}$ be the dual base of $T^{*} M . r$ acts as a derivation $r_{d}$ of $\Lambda^{(0, .)}\left(T^{*} M\right)$ by the formula

$$
r_{d}=-\sum r\left(w_{i}, \bar{w}_{j}\right) \bar{w}^{j} \wedge i_{\bar{w}_{i}} .
$$

Also we identify the two form $r$ with the self-adjoint matrix $r \in$ End $\overline{T M}$ such that if $X, Y \in T M$, then

$$
r(X, \bar{Y})=\langle X, \dot{r} \bar{Y}\rangle
$$

For $0 \leqq q \leqq l$, let $r_{d}^{q}$ be the restriction of $r_{d}$ to $\Omega^{(0, q)}\left(\mu^{\otimes p} \otimes \xi \otimes E_{p}\right)$. As $t \downarrow 0$, we have the asymptotic expansion

$$
(2 \pi)^{-l} \int_{M} \frac{(\operatorname{det} \stackrel{\circ}{r}) \operatorname{Tr}\left[e^{t r_{d}^{q}}\right]}{\operatorname{det}\left(I-e^{-t r}\right)} d x=\sum_{j=-l}^{k} a_{j}^{q} t^{j}+o\left(t^{k}\right)
$$

Theorem 2. For any $t>0,0 \leqq q \leqq l$,

$$
\lim _{p \rightarrow+\infty} \frac{1}{r k\left(E_{p}\right)} p^{-l} \operatorname{Tr}\left[\exp \left(-\frac{t}{p} \bar{\square}_{p}^{q}\right)\right]=(2 \pi)^{-l} r k(\xi) \int_{M} \frac{\operatorname{det}\left(r^{\circ}\right) \operatorname{Tr}\left[e^{t r_{d}^{q}}\right]}{\operatorname{det}\left(I-e^{-t r^{\circ}}\right)} d x,
$$

and the convergence in (18) is uniform as t varies in compact subsets of $R_{+}^{*}$. Moreover for any $j \geqq-l, 0 \leqq q \leqq l$, as $p \rightarrow+\infty$

$$
a_{p, j}^{q}=r k(\xi) a_{j}^{q}+O\left(\frac{1}{\sqrt{p}}\right) .
$$

In (14), o $\left(t^{k}\right)$ is uniform with respect to $p \in N$. Finally there exist $C>0, c>0, c^{\prime}>0$ such that for any $p \in N, 1 \leqq q \leqq l, t \geqq 1$,

$$
\frac{p^{-l}}{r k\left(E_{p}\right)} \operatorname{Tr}\left[\exp \left(-\frac{t}{p} \bar{\square}_{p}^{q}\right)\right] \leqq C \exp \left(-\left(c-\frac{c^{\prime}}{p}\right) t\right) \text {. }
$$


Proof. If $E_{p}$ does not depend on $p,(18)$ is a consequence of [B1, Theorem 1.5]. We now briefly show how to modify the arguments in the proof of [B1, Theorem 1.5] to obtain (18) in full generality.

The first difficulty is to prove the analogue of [B1, Eqs. (1.34)-(1.36)] so that the asymptotic representation in [B1, Eq. (1.37)] can be used. Since $E_{p}$ is flat, if $P_{p, t}^{q}\left(x, x^{\prime}\right)$ is the smooth kernel for $\exp \left(-t \bar{\square}_{p}^{q}\right)$, by [B1, Eq. (1.34)], $E_{p}$ only contributes to $\operatorname{Tr}\left[P_{p, t}^{q}(x, x)\right]$ by a unitary parallel transport operator from $E_{p, x}$ into $E_{p, x}$ along loops starting and ending at $x$. The absolute value of the trace of this operator is dominated by $r k\left(E_{p}\right)$. When dividing $\operatorname{Tr}\left[P_{p, t}^{q}(x, x)\right]$ by $r k\left(E_{p}\right)$, we are essentially back to the situation considered in [B1, Theorem 1.5], i.e. the problem of calculating the asymptotics of $\frac{p^{-l}}{r k\left(E_{p}\right)} \operatorname{Tr}\left[P_{p, t}^{q}(x, x)\right]$ can be localized in an arbitrarily small neighborhood of $x$. The proof of (18) then proceeds as in [B1, Theorem 1.5].

Let $\nabla^{T M}$ be the holomorphic Hermitian connection on $\left(T M,\|\|_{T M}\right)$. All the vector bundles which we now consider are equipped with holomorphic Hermitian connections.

Since the $a_{j, p}^{q}$ do not depend on $E_{p}$, we may and we will assume that $E_{p}$ is the trivial Hermitian line bundle on $M$. To make our formulas simpler, we will also assume that the Hermitian metric \|\|$_{T M}$ on $T M$ is Kähler.

Let $\Delta_{p}$ be the horizontal (or Bochner) Laplacian acting on $\Omega^{(0, .)}\left(\mu^{\otimes p} \otimes \xi\right.$ ). Take $w_{1}, \ldots, w_{l}$ as in (15). By Lichnerowicz's formula [B1, Proposition 1.2], we know that

$$
\begin{gathered}
\bar{\square}_{p}=\frac{-\Delta_{p}}{2}-\bar{w}^{i} \wedge i_{\bar{w}_{j}}\left(L^{\xi}+\frac{1}{2} \operatorname{Tr}\left[R^{T M}\right] I_{\xi}\right)\left(\bar{w}_{i}, w_{j}\right)-\frac{1}{2} L^{\xi}\left(w_{i}, \bar{w}_{i}\right) \\
-p r^{d}-\frac{p r}{2}\left(w_{i}, \bar{w}_{i}\right) .
\end{gathered}
$$

Take $x \in M$. Using geodesic coordinates, we may and we will identify an open neighborhood $U$ of $x$ in $M$ with the open ball $B(0, \varepsilon)$ of center 0 and radius $\varepsilon>0$ in $C^{l}$ (with $x$ identified to $0 \in C^{l}$ ). We also trivialize the vector bundles $\Lambda^{(0, .)}\left(T^{*} M\right)$, $\xi$ and $\mu$ by parallel transport along the radial lines in $U \simeq B(0, \varepsilon)$. Let $Y=\left(Y^{1}, \ldots, Y^{2 l}\right)$ be real coordinates in $C^{l} \simeq R^{2 l}$.

Let $\left(e_{1}, \ldots, e_{2 l}\right)$ be the real orthonormal frame in $T_{R} M$ obtained by parallel transport along radial lines from a given orthonormal frame in $\left(T_{R} M\right)_{x}$. Then if $\nabla_{p}$ is the holomorphic Hermitian connection on $\Lambda^{(0, .)}\left(T^{*} M\right) \otimes \mu^{\otimes p} \otimes \xi$, we have the identity

$$
\Delta_{p}=\sum_{1}^{2 l}\left(\nabla_{p, e_{i}}\right)^{2}-\sum_{1}^{2 l} \nabla_{p, \nabla_{e_{i}}^{T M} e_{i}} .
$$

Let $\Gamma, A$ be the connection forms of the holomorphic Hermitian connections on $\Lambda^{(0, .)}\left(T^{*} M\right) \otimes \xi$ and $\mu$ with respect to the considered trivializations. Then if $\frac{\partial}{\partial e_{i}}$ is the differentiation operator in the direction $e_{i}$, we have

$$
\nabla_{p, e_{i}}=\frac{\partial}{\partial e_{i}}+\Gamma_{e_{i}}+p A_{e_{i}} .
$$


Remenber that $A(Y)$ vanishes at $Y=0$. Also since $\mu$ is trivialized by parallel transport along straight lines starting at 0 , for $Y \in B(0, \varepsilon), A_{Y}(Y)=0$. We then find easily that for any $Y \in B(0, \varepsilon)$, as $p \rightarrow+\infty$,

$$
\sqrt{p} A \cdot\left(\frac{Y}{\sqrt{p}}\right) \rightarrow \frac{1}{2} r_{x}(Y, .)
$$

We now express the operator $\bar{\square}_{p}$ in the coordinates $Y$ using the previously described trivializations of $\Lambda^{(0,1)}\left(T^{*} M\right), \mu$ and $\xi$. Let $\bar{\square}_{p, 1}$ be the operator obtained by making the change of variables $Y \rightarrow \sqrt{p} Y$ in $\bar{\square}_{p}$.

Using (22)-(24), we find easily that as $p \rightarrow+\infty$,

$$
\frac{1}{p} \bar{\square}_{p, 1} \rightarrow \mathscr{L}_{x}=-\frac{1}{2} \sum_{1}^{2 l}\left(\frac{\partial}{\partial e_{i}}+\frac{1}{2} r_{x}\left(Y, e_{i}\right)\right)^{2}-r_{x}^{d}-\sum_{1}^{l} \frac{r_{x}}{2}\left(w_{i}, \bar{w}_{i}\right)
$$

in the sense that the smooth coefficients of the second order operator $\frac{1}{p} \bar{\square}_{p, 1}$, converge uniformly on compact sets in $C^{l} \simeq R^{2 l}$ to the corresponding coefficients of the operator $\mathscr{L}_{x}$. More precisely, $\frac{1}{p} \bar{\square}_{p, 1}-\mathscr{L}_{x}$ is of order $\frac{1}{\sqrt{p}}$, i.e., for any $k \in N$, the norms of the derivatives of order $\leqq k$ of the coefficients of $\frac{1}{p} \bar{\square}_{1, p}-\mathscr{L}_{x}$ are dominated on compact sets by $\frac{C}{\sqrt{p}}$.

Now by Seeley [S], Greiner [Gre], we know that the coefficients in the small $t$ expansion of the heat kernel on the diagonal associated with an elliptic operator depend smoothly on the local symbol of the considered operator. We then deduce (19) from the previous considerations. The fact that in (14), $o\left(t^{k}\right)$ is uniform with respect to $p$ also follows from the previous arguments.

With the notations of Theorem 1 , for $t \geqq 1$, we have

$$
\operatorname{Tr}\left[\exp \left(-\frac{t}{p} \bar{\square}_{p}^{q}\right)\right] \leqq \operatorname{Tr}\left[\exp \left(-\frac{\bar{\square}_{p}^{q}}{p}\right)\right] \exp \left(-\frac{(t-1)}{p} \lambda_{p}^{q}\right) .
$$

By (18), as $p \rightarrow+\infty, \frac{p^{-l}}{r k\left(E_{p}\right)} \operatorname{Tr}\left[\exp \left(-\frac{\bar{\square}_{p}^{q}}{p}\right)\right]$ has a finite limit. Also by Theorem 1, there exist $c>0, c^{\prime}>0$ such that for $p \in N, q \geqq 1, t \geqq 1$,

$$
\exp \left(-\frac{(t-1)}{p} \lambda_{p}^{q}\right) \leqq \exp \left(-(t-1)\left(c-\frac{c^{\prime}}{p}\right)\right)
$$

(20) follows from (26), (27).

When the metric on $T M$ is not Kähler, as explained in [B1, proof of Theorem 1.5], the formula for $\bar{\square}_{p}$ in (21) is more complicated, but all the extra terms are killed by the factor $\frac{1}{p}$. Details are left to the reader. 
Remark 3. Of course (18) can also be derived from (25). An analogue of (25) has been used by Getzler [Ge] to derive an extension of (18) for Heisenberg manifolds.

For any $p \in N, k \in N$, as $t \downarrow 0$ we have the asymptotic expansion

$$
\frac{p^{-l}}{r k\left(E_{p}\right)} \sum_{q=1}^{l}(-1)^{q} q \operatorname{Tr}\left[\exp \left(\frac{-t}{p} \bar{\square}_{p}^{q}\right)\right]=\sum_{j=-l}^{k} b_{p, j} t^{j}+o\left(t^{k}\right) \text {. }
$$

In (28), the $b_{p, j}$ do not depend on $E_{p}$. Also as $t \downarrow 0$,

$$
(2 \pi)^{-l} \int_{M} \operatorname{det}(\stackrel{\circ}{r}) \operatorname{Tr}\left[\left(I-e^{i r}\right)^{-1}\right] d x=\frac{b-1}{t}+b_{0}+\ldots+b_{k} t^{k}+o\left(t^{k}\right) .
$$

Remember that $\omega$ is the Kähler form of the manifold $M . \omega$ is given by Eq. (1). $\exp \left(\frac{-r}{2 i \pi}\right)$ denotes the exponential in $\Lambda\left(T_{R}^{*} M\right)$ of the two form $\left(\frac{-r}{2 i \pi}\right)$.

Theorem 4. For any $t>0$,

$$
\begin{gathered}
\lim _{p \rightarrow+\infty} \frac{1}{r k\left(E_{p}\right)} p^{-l} \sum_{1}^{l}(-1)^{q} q \operatorname{Tr}\left[\exp \left(-\frac{t}{p} \bar{\square}_{p}^{q}\right)\right] \\
=(2 \pi)^{-l} r k(\xi) \int_{M}(\operatorname{det} r) \operatorname{Tr}\left[\left(I-e^{t r}\right)^{-1}\right] d x,
\end{gathered}
$$

and the convergence is uniform as $t$ varies in compact subsets of $R_{+}^{*}$.

As $p \rightarrow+\infty$,

$$
b_{p, j}=0\left(\frac{1}{\sqrt{p}}\right) ; j \leqq-2, \quad b_{p, j}=r k(\xi) b_{p}+0\left(\frac{1}{\sqrt{p}}\right) ; j \geqq-1 .
$$

The following identities hold

$$
b_{-1}=\int_{M} \frac{\omega}{2 \pi} \exp \left(\frac{-r}{2 i \pi}\right), \quad b_{0}=\frac{l}{2} \int_{M} \exp \left(\frac{-r}{2 i \pi}\right) .
$$

In (28), for any $k \in N, o\left(t^{k}\right)$ is uniform with respect to $p$. There exists $C>0, c>0$, $c>0$, such that for any $p \in N, t \geqq 1$,

$$
\left|\frac{p^{-l}}{r k\left(E_{p}\right)} \sum_{q=1}^{l}(-1)^{q} q \operatorname{Tr}\left[\exp \left(\frac{-t \bar{\square}_{p}^{q}}{p}\right)\right]\right| \leqq C \exp \left(-\left(c-\frac{c^{\prime}}{p}\right) t\right) .
$$

Proof. Note the identity

$$
\sum_{q=1}^{l}(-1)^{q} q \operatorname{Tr}\left[e^{t r_{d}^{q}}\right]=\frac{\partial}{\partial b}\left[\operatorname{det}\left(I-b e^{-t_{r}^{\circ}}\right)\right]_{b=1} .
$$

From (34), we deduce that

$$
\frac{\operatorname{det}(\stackrel{\circ}{r})}{\operatorname{det}\left(I-e^{-t_{r}^{\circ}}\right)} \sum_{q=1}^{l}(-1)^{q} q \operatorname{Tr}\left[e^{t r_{d}^{q}}\right]=(\operatorname{det} \stackrel{\circ}{r}) \operatorname{Tr}\left[\left(I-e^{t \stackrel{\circ}{r}}\right)^{-1}\right] .
$$


Then (30) follows from (18), (35). Equation (31) is a consequence of (19). Note the equalities of $(l, l)$ form

$$
\operatorname{det}\left(\frac{\dot{r}}{2 \pi}\right) d x=\frac{\left(-\frac{r}{2 i \pi}\right)^{l}}{l !}
$$

Equation (32) is then a straightforward calculation, which is left to the reader. Equation (33) is a consequence of (20).

Remark 5. Assume temporarily that $\omega$ is closed, i.e. $(M, \omega)$ is Kähler. By [BGS1, Theorem 2.16], we know that for $j \leqq-2 . b_{p, j}=0$. If $R^{M}$ is the curvature of the holomorphic Hermitian connection on $T M$, by [BGS1, Theorem 2.16], we also know that if $T d$ is the Todd polynomial given by the formula $T d(x)=\frac{x}{1-e^{-x}}$, then

$$
\begin{aligned}
b_{p,-1}= & p^{-l+1} \int_{M} \frac{\omega}{2 \pi} T d\left(\frac{-R^{M}}{2 i \pi}\right) \operatorname{Tr}\left(\exp \left(\frac{-L^{\xi}}{2 i \pi}\right)\right] \exp \left(\frac{-p r}{2 i \pi}\right), \\
b_{p, 0}= & p^{-l} \int_{M}\left\{\frac{\partial}{\partial b}\left[T d\left(\frac{-R^{M}}{2 i \pi}-b I\right)\right]_{b=0}+l T d\left(\frac{-R^{M}}{2 i \pi}\right)\right\} \\
& \cdot \operatorname{Tr}\left[\exp \left(\frac{-L^{\xi}}{2 i \pi}\right)\right] \exp \left(\frac{-p r}{2 i \pi}\right) .
\end{aligned}
$$

Note that with the notations of [BGS1], $D^{2}=2 \bar{\square}_{p}$, which accounts for the discrepancy by a factor 2 in $b_{p,-1}$ with respect to [BGS1, Theorem 2.16].

Using the expansion $T d(x)=1+\frac{x}{2}+\ldots$, one easily verifies that (36) is compatible with (31), (32). It is remarkable that asymptotically, (36) is still true, even if $(M, \omega)$ is not Kähler.

\section{d) The Asymptotics of the Analytic Torsion}

By Theorem 1, there exists $p_{0} \in N$ such that for $p \geqq p_{0}, 1 \leqq q \leqq l$, the operator $\bar{\square}_{p}^{q}$ is invertible.

Definition 6. For $p \geqq p_{0}, \operatorname{Re}(s)>l$, set

$$
\zeta_{p}(s)=-\frac{1}{\Gamma(s)} \int_{0}^{+\infty} t^{s-1}\left(\sum_{1}^{l}(-1)^{q} q \operatorname{Tr}\left[\exp \left(-t \bar{\square}_{p}^{q}\right)\right]\right) d t .
$$

By a well-known result of Seeley $[\mathrm{Se}], \zeta_{p}(s)$ extends into a meromorphic function of $s$, which is holomorphic at $s=0$. By definition $\exp \left(-\zeta_{p}^{\prime}(0)\right)$ is the analytic torsion of Ray-Singer [RS] for the vector bundle $\mu^{\otimes p} \otimes \xi \otimes E_{p}$.

Remark 7. If we were to follow the conventions of [BGS1,2], in (37), we should replace $\operatorname{Tr}\left[\exp \left(-t \bar{\square}_{p}^{q}\right)\right]$ by $\operatorname{Tr}\left[\exp \left(-2 t \bar{\square}_{p}^{q}\right)\right]$, which is equivalent to scaling the metric on $T M$ by a factor $\frac{1}{2}$.

We now prove the main result of this paper. 
Theorem 8. As $p \rightarrow+\infty$, then

$$
\zeta_{p}^{\prime}(0)=r k\left(E_{p}\right) r k(\xi)\left\{\frac{1}{2} \int_{M} \log \left(\operatorname{det}\left(\frac{p \dot{\circ}}{2 \pi}\right)\right) \exp \left(\frac{-p r}{2 i \pi}\right)+o\left(p^{l}\right)\right\} .
$$

Proof. Set

Clearly

$$
\tilde{\zeta}_{p}(s)=-\frac{1}{\Gamma(s)} \int_{0}^{+\infty} p^{-l} \frac{t^{s-1}}{r k\left(E_{p}\right)} \sum_{q=1}^{l}(-1)^{q} q \operatorname{Tr}\left[\exp \left(\frac{-t}{p} \bar{\square}_{p}^{q}\right)\right] d t .
$$

and so

$$
\frac{p^{-l}}{r k\left(E_{p}\right)} \zeta_{p}(s)=p^{-s} \tilde{\zeta}_{p}(s)
$$

$$
\frac{p^{-l}}{r k\left(E_{p}\right)} \zeta_{p}^{\prime}(0)=-\log (p) \tilde{\zeta}_{p}(0)+\tilde{\zeta}_{p}^{\prime}(0) .
$$

Also with the notations in (28), we have

$$
\tilde{\zeta}_{p}(0)=-b_{p, 0}
$$

By Theorem 4 , as $p \rightarrow+\infty$,

$$
b_{p, 0}=r k(\xi) \frac{l}{2} \int_{M} \operatorname{det}\left(\frac{\stackrel{r}{2 \pi}}{2 \pi}\right) d x+0\left(\frac{1}{\sqrt{p}}\right) .
$$

Also we have the obvious identity

$$
\begin{aligned}
\widetilde{\zeta}_{p}^{\prime}(0)= & -\int_{0}^{1}\left\{\frac{p^{-l}}{r k\left(E_{p}\right)}\left(\sum_{q=1}^{l}(-1)^{q} q \operatorname{Tr}\left[\exp \left(\frac{-t}{p} \bar{\square}_{p}^{q}\right)\right]-\sum_{j=-l}^{1} b_{p, j} t^{j}\right\} \frac{d t}{t}\right. \\
& -\int_{1}^{+\infty}\left\{\frac{p^{-l}}{r k\left(E_{p}\right)} \sum_{q=1}^{l}(-1)^{q} q \operatorname{Tr}\left[\exp \left(\frac{-t}{p} \bar{\square}_{p}^{q}\right)\right]\right\} \frac{d t}{t}-\sum_{\substack{j=-l \\
j \neq 0}}^{1} \frac{b_{p, j}}{j}+\Gamma^{\prime}(1) b_{p, 0} .
\end{aligned}
$$

By Theorem 4, we know that there exists $C>0$ such that for any $p \in N, t \in] 0,1]$,

$$
\left|\frac{p^{-l}}{r k\left(E_{p}\right)}\left(\sum_{q=1}^{l}(-1)^{q} q \operatorname{Tr}\left[\exp \left(\frac{-t}{p} \bar{\square}_{p}^{q}\right)\right]\right)-\sum_{j=-l}^{1} b_{p, j} t^{j}\right| \leqq C t .
$$

Using (29), (33), (44) and dominated convergence, we find that as $p \rightarrow+\infty$,

$$
\begin{aligned}
\tilde{\zeta}_{p}^{\prime}(0) \rightarrow \eta= & -r k(\xi) \int_{0}^{1}\left\{(2 \pi)^{-l} \int_{M} \operatorname{det}\left(r^{\circ}\right) \operatorname{Tr}\left[\left(I-e^{t r}\right)^{-1}\right] d x-\frac{b-1}{t}-b_{0}-b_{1} t\right\} \frac{d t}{t} \\
& -r k(\xi) \int_{1}^{+\infty}\left\{(2 \pi)^{-l} \int_{M} \operatorname{det}(\stackrel{\circ}{r}) \operatorname{Tr}\left[\left(I-e^{t r}\right)^{-1}\right] d x\right\} \frac{d t}{t} \\
& +r k(\xi)\left(b_{-1}-b_{1}+\Gamma^{\prime}(1) b_{0}\right) .
\end{aligned}
$$

For $s \in C, \operatorname{Re}(s)>1$, set

$$
\tilde{\zeta}(s)=\frac{-1}{\Gamma(s)} \int_{0}^{+\infty} t^{s-1}\left\{(2 \pi)^{-l} \int_{M} \operatorname{det}(\stackrel{\circ}{r}) \operatorname{Tr}\left[\left(I-e^{t r}\right)^{-1}\right] d x\right\} d t
$$


It is clear that the function $\tilde{\zeta}(s)$ extends into a meromorphic function of $s$, which is holomorphic at $s=0$. One then easily verifies that

$$
\eta=(r k \xi) \tilde{\zeta}^{\prime}(0),
$$

Also since $\dot{r}$ considered as an element of End $(\overline{T M})$ has positive eigenvalues, we find that for $\operatorname{Re}(s)>1$,

$$
\tilde{\zeta}(s)=(2 \pi)^{-l}\left(\int_{M} \operatorname{det}\left(r^{\circ}\right) \operatorname{Tr}\left[\dot{r}^{-s}\right] d x\right) \frac{1}{\Gamma(s)} \int_{0}^{+\infty} t^{s-1} \frac{e^{-t}}{1-e^{-t}} d t .
$$

Let $\zeta_{R}(s)=\sum_{1}^{+\infty} \frac{1}{n^{s}}$ be the Riemann zeta function. Classically, for $\operatorname{Re}(s)>1$,

$$
\zeta_{R}(s)=\frac{1}{\Gamma(s)} \int_{0}^{+\infty} t^{s-1} \frac{e^{-t}}{1-e^{-t}} d t
$$

So from (48), (49), we find that

$$
\tilde{\zeta}(s)=(2 \pi)^{-l}\left(\int_{M}\left(\operatorname{det} r^{\circ}\right) \operatorname{Tr}\left[\dot{r}^{-s}\right] d x\right) \zeta_{R}(s) .
$$

From (50), we deduce that

$$
\tilde{\zeta}^{\prime}(0)=-(2 \pi)^{-l} \int_{M} \operatorname{det}\left(\stackrel{\circ}{)} \operatorname{Tr}\left[\log r^{\circ}\right] d x \zeta_{R}(0)+(2 \pi)^{-l} l \int_{M} \operatorname{det}\left(r^{\circ}\right) d x \zeta_{R}^{\prime}(0) .\right.
$$

Also we know that

$$
\zeta_{R}(0)=-\frac{1}{2} ; \zeta_{R}^{\prime}(0)=-\frac{1}{2} \log (2 \pi) .
$$

So from (51), (52), we get

$$
\tilde{\zeta}^{\prime}(0)=\frac{1}{2} \int_{M} \operatorname{det}\left(\frac{\dot{r}}{2 \pi}\right) \log \left(\operatorname{det}\left(\frac{\dot{r}}{2 \pi}\right)\right) d x .
$$

Equation (38) now follows from (40), (42) and from (45), (47), (53).

Remark 9. In [F, Theorem 2], Faltings gives an asymptotic estimate for the ratio of the Faltings metric to the $L^{2}$ metric of the determinant of the cohomology of a line bundle on a Riemann surface. In view of a result of Bost [Bo], which compares the Faltings metric and the Quillen metric, Theorem 8 provides a sharper estimate than [F, Theorem 2].

\section{e) Asymptotic Anomaly Formulas for the $L_{2}$ Metric}

Let now \|\|$_{T M}$ and \|\|$_{\tilde{T}_{M}}$ be two Hermitian metrics on TM. We otherwise keep the metrics on $\mu, \xi, E_{p}$ fixed.

Set

$$
\lambda_{p}=\operatorname{det}\left(H^{0}\left(M ; \mu^{\otimes p} \otimes \xi \otimes E_{p}\right)\right) .
$$

As a subspace of the set of square-integrable sections of $\mu^{\otimes p} \otimes \xi \otimes E_{p}$, the vector space $H^{0}\left(M, \mu^{\otimes p} \otimes \xi \otimes E_{p}\right)$ inherits two Hermitian metrics associated with the 
metrics \|\|$_{T M}$ and \|\|$\tilde{T M}$ respectively, and with the given metrics on $\mu, \xi$ and $E_{p}$. Let ||$_{p}$ and ||$_{p}$ be the corresponding metrics on $\lambda_{p}$.

Theorem 10. As $p \rightarrow+\infty$, then

$$
\log \left(\frac{||_{p}^{2}}{||_{p}^{2}}\right)=r k\left(E_{p}\right) r k(\xi)\left[p^{l} \int_{M} \log \left(\operatorname{det} \frac{\|\|_{T M}^{2}}{\|\|_{T M}^{2}}\right) \exp \left(\frac{-r}{2 i \pi}\right)+o\left(p^{l}\right)\right] .
$$

Proof. Let $\zeta_{p}^{\prime}(0)$ and $\tilde{\zeta}_{p}^{\prime}(0)$ be the real numbers which appear in Theorem 8 respectively associated with the metrics \|\|$_{T M}$ and \|\|$\tilde{T}_{M}$ and the given metrics on $\mu, \xi$ and $E_{p}$. By Theorem 8, we find that as $p \rightarrow+\infty$,

$$
\tilde{\zeta}_{p}^{\prime}(0)-\zeta_{p}^{\prime}(0)=-r k\left(E_{p}\right) r k(\xi)\left\{\frac{p^{l}}{2} \int_{M} \log \left(\operatorname{det} \frac{\|\|_{T M}^{2}}{\|\|_{T M}^{2}}\right) \exp \left(\frac{-r}{2 i \pi}\right)+o\left(p^{l}\right)\right\} .
$$

For $p$ large enough, for $q \geqq 1, H^{q}\left(M ; \mu^{\otimes p} \otimes \xi \otimes E_{p}\right)=0$, and $\lambda_{p}$ coincides with the determinant line of the cohomology of $\mu^{\otimes p} \otimes \xi \otimes E_{p}$.

Let \|\|$_{p}$ and \|\|$_{p}$ be the Quillen metrics on $\lambda_{p}^{\prime}=\lambda_{p}^{-1}$ constructed in [BGS2] respectively associated with the metrics \|\|$_{T M}$ and \|\|$\tilde{T}_{M}$ and the given metrics on $\mu, \xi$, and $E_{p}$. Assume first that \|\|$_{T M}$ and \|\|$\tilde{r}_{M}$ are both Kähler metrics. Then by [BGS2, Theorem 1.22], we find easily that as $p \rightarrow+\infty$,

$$
\log \frac{\|\|_{p}^{2}}{\|\|_{p}^{2}}=r k\left(E_{p}\right) r k(\xi)\left\{-\frac{p^{l}}{2} \int_{M} \log \left(\operatorname{det}\left(\frac{\|\|_{T M}^{2}}{\|\|_{T M}^{2}}\right)\right) \exp \left(\frac{-r}{2 i \pi}\right)+o\left(p^{l}\right)\right\} .
$$

Also by definition of the Quillen metric, we know that

$$
\log \left(\frac{\|\|_{p}^{2}}{\|\|_{p}^{2}}\right)=-\widetilde{\zeta}_{p}^{\prime}(0)+\zeta_{p}^{\prime}(0)-\log \left(\frac{||_{p}^{2}}{||_{p}^{2}}\right)
$$

Equation (55) then follows from (56)-(58).

If the metrics \|\|$_{T M}$ or \|\|$\tilde{T}_{M}$ are not Kähler, using [BGS2, Theorem 1.18] (which is valid for any metric) and the methods of the proof of Theorem 2 (which permit us to calculate explicitly the asymptotics as $p \rightarrow+\infty$ of the terms appearing in small time expansions of traces of heat kernels), we find easily that formula (57) holds in full generality. Our theorem is proved.

\section{f) The Asymptotics of the Analytic Torsion of a Positive Line Bundle with an Arbitrary Metric}

We now assume that \|\|$_{\mu}$ is an arbitrary smooth metric on the positive line bundle $\mu$. If $\tilde{\nabla}^{\mu}$ is the corresponding holomorphic Hermitian connection on $\mu$, and if $\tilde{r}$ is the curvature of $\tilde{\nabla}^{\mu}$, we do not assume any more that $\tilde{r}$ is positive.

Take $p_{0}$ as in Definition 6 . Then for $p \geqq p_{0}$, we can define the function $\tilde{\zeta}_{p}(s)$ associated with the metric \|\|$_{T M}$ on $T M$, with the metric \|\|$\tilde{\mu}$ on $\mu$, and with the flat vector bundle $E_{p}$. 
The main difference with the situation considered in Sects. a)-e) is that Theorem 1 does not hold any more for the new Laplacian. Still we prove the following estimate.

Theorem 11. There exists $C>0$ such that for $p \geqq p_{0}$,

$$
\left|\widetilde{\zeta}_{p}^{\prime}(0)\right| \leqq C r k\left(E_{p}\right) p^{l+1}
$$

Proof. We use the notations of the previous sections, the notation $\sim$ now refers to the new metric \|\|$_{\mu}$. There exist constants $d, D, 0<d<D<+\infty$, such that

$$
d \leqq\left(\frac{\|\|_{\mu}}{\|\|_{\mu}}\right)^{2} \leqq D
$$

Now by proceeding as in Bismut [B2, Proposition 3.3] we find that if $a=d / D$, for $p \in N, 0 \leqq q \leqq \ell$

$$
\tilde{\lambda}_{p}^{q} \geqq a^{p} \lambda_{p}^{q}
$$

The identity (18) is still valid with respect to the new metric \|\|$\sim$.

Using Theorem 1 and (18), we find that instead of (20), we now have the bound for $p \in N, 1 \leqq q \leqq \ell, t \geqq 1$,

$$
\frac{p^{-\ell}}{r k\left(E_{p}\right)} \operatorname{Tr}\left[\exp \left(\frac{-t}{p} \overline{\tilde{\square}}_{p}^{q}\right)\right] \leqq C \exp \left(-\left(c-\frac{c^{\prime}}{p}\right) a^{p} t\right) .
$$

Note that $0<a<1$. Then if $p \rightarrow+\infty$,

$$
\int_{1}^{+\infty} \exp \left(\frac{-c}{2} a^{p} t\right) \frac{d t}{t} \simeq-p \log a .
$$

Equation (59) follows easily from the analogue of (18) and from (63).

\section{References}

[B1] Bismut, J.M.: Demailly's asymptotic Morse inequalities: a heat equation proof. J. Funct. Anal. 72, 263-278 (1987)

[B2] Bismut, J.M.: Superconnection currents and complex immersions. Invent. Math. (to appear)

[BGS1] Bismut, J.M., Gillet, H., Soulé, C.: Analytic torsion and holomorphic determinant bundles. II. Direct images and Bott-Chern forms. Commun. Math. Phys. 115, 79-126 (1988)

[BGS2] Bismut, J.M., Gillet, H., Soulé, C.: Analytic torsion and holomorphic determinant bundles. III. Quillen metrics on holomorphic determinants. Commun. Math. Phys. 115, 301-351 (1988)

[BV] Bismut, J.M., Vasserot, E.: Comportement asymptotique de la torsion analytique associée aux puissances d'un fibré en droites positif. C.-R. Acad. Sci. Paris 307, 779-781 (1988)

[Bo] Bost, J.B.: To appear

[D1] Demailly, J.P.: Champs magnétiques et inégalités de Morse pour la $d^{\prime \prime}$ cohomologie. Ann. Inst. Fourier 35, 189-229 (1985)

[D2] Demailly, J.P.: Sur l'identité de Bochner-Kodairo-Nakano en géométrie Hermitienne. Séminaire Dolbeault-Lelong-Skoda. Lecture Notes in Math., vol. 1198, pp. 88-97. Berlin, Heidelberg, New York: Springer 1986 
[F] Faltings, G.: Calculus on arithmetic surfaces. Ann. Math. 119, 387-424 (1984)

[Ge] Getzler, E.: An analogue of Demailly's inequality for strictly pseudoconvex manifolds. J. Diff. Geom. 29, 231-244 (1989)

[GS] Gillet, H., Soulé, C.: Amplitude arithmétique. C.-R. Acad. Sc. Paris 307, 887-890 (1988)

[GrH] Griffiths, P., Harris, J.: Principles of algebraic geometry. New York: Wiley 1978

[Gre] Greiner, P.: An asymptotic expansion for the heat equation. Arch. Ration. Mech. Anal. 41, 163-218 (1971)

[Q] Quillen, D.: Determinants of Cauchy-Riemann operators. Funct. Anal. Appl. 44, 31-34 (1985)

[RS] Ray, D.B., Singer, I.M.: Analytic torsion for complex manifolds. Ann. Math. 98, 154-177 (1973)

[S] Seeley, R.T.: Complex powers of an elliptic operator. Proc. Symp. Pure Math., Vol. 10, pp. 288-307. Providence, R.I: Am. Math. Soc. 1967

[V] Vojta, P.: An extension of the Thue-Siegel-Dyson-Gel'fond theorem. Preprint 1989

Communicated by A. Jaffe

Received October 19, 1988; in revised form March 28, 1989 
